\title{
Peer-to-peer File-sharing over Mobile Ad hoc Networks ${ }^{1}$
}

\author{
Gang Ding and Bharat Bhargava \\ Department of Computer Sciences \\ Purdue University \\ West Lafayette, IN 47907, USA \\ \{dingg,bb\}@cs.purdue.edu
}

\begin{abstract}
Current peer-to-peer file-sharing systems mostly work on wired networks. Mobile ad hoc network is characterized as multi-hop wireless communications between mobile devices. In this paper, five routing approaches with different complexity are proposed to enable peer-to-peer file-sharing over mobile ad hoc networks. The complexity of the proposed approaches is evaluated and compared. It is concluded that the cross-layer protocols perform better than simply overlaying peer-to-peer searching protocol on mobile ad hoc networks.
\end{abstract}

\section{Introduction}

Peer-to-peer (P2P) system aims to share information among a large number of users without assistance of explicit servers. It has attracted more and more researchers in recent years, motivated by the popularity of file-sharing applications over internet, such as Napster and Gnutella ([1]). There are many research issues on P2P networks, among which the searching (or application layer routing) algorithm is the central topic. Napster uses a central server to maintain index of all information so that every peer should contact the server to lookup the information. Gnutella works in a pure P2P way where every peer broadcasts its query to all its neighbors. Peers form an overlay topology which might be far different from the underlying physical network topology. But this kind of query 'flood' takes too much network bandwidth so that scalability issue arises. Some highly structured

\footnotetext{
${ }^{1}$ This work is support by NSF grants ANI 0219110 and CCR 0001788.
}

P2P lookup algorithms have been recently proposed, such as Chord, Pastry, Tapestry and CAN ([2]). They all employ a similar technique named Distributed Hash Table (DHT), in which every file and peer is assigned a unique key by a hash function. The keys, along with the network address of the peer storing the corresponding files, are evenly distributed among all participating peers. Each peer maintains a routing table and queries are only directed to those peers in the routing table. For Chord, Pastry and Tapestry, the routing table size is $O(\log n)$ and the searching path length is $O(\log n)$, where $n$ is the total number of files and peers. For CAN, peers have routing table size of $O(d)$ and searching path length of $O\left(d n^{1 / d}\right)$. The fast and accurate searching ability of DHT-based algorithms is very attractive and have been applied to different kinds of applications in wired networks.

Mobile ad hoc network (MANET) is another popular research area due to the rapid progress of mobile wireless communications. MANET is characterized as an infrastructure-less mobile wireless network, in which two mobile nodes communicate with each other through intermediate nodes. Since there is no explicit server, every mobile node should work autonomously. One of the main research issues in MANET is network routing ([3]). In proactive (or table-driven) routing protocols, such as DSDV, every mobile node tries to maintain a routing table involving the complete information of network topology. But this needs a lot of computation efforts and communication bandwidth to maintain the accurate routing tables. On the other hand, the reactive (or ondemand) routing protocols, such as DSR and AODV, try to find a route to the destination only when it is necessary. The route request is broadcasted throughout 
the whole network, and the route response is returned when a mobile node knows the route to the requested destination or itself is the destination.

We notice that there are some similar features for both P2P file-sharing networks and MANET ([4]). If we also call a mobile node in MANET a mobile peer, (a) there is no peer in either P2P network or MANET that acts explicitly as a central server, and every peer should collaborate with other peers in order to make the whole system work. (b) The major problem in $\mathrm{P} 2 \mathrm{P}$ and MANET is how to find the requested data or route efficiently. (c) The topology of P2P and MANET is changing frequently because of peer on-off or mobility. (d) In both systems, flooding or broadcasting is employed to some extent in order to exchange data or routing information among different peers, which raises the scalability problem. There are also some differences between P2P and MANET. For example, (a) P2P refers to the application layer in the protocol stack, while MANET focuses on the network and lower layers. (b) The peers in MANET are mobile and constrained by limited energy, bandwidth and computation power, which is not a big concern in $\mathrm{P} 2 \mathrm{P}$ file-sharing systems over internet. (c) For the execution of broadcast, a $\mathrm{P} 2 \mathrm{P}$ overlay is a single cast network which only generates virtual broadcast consisting of a number of single cast messages. In contrast, MANET always performs a physical broadcast.

The above similarities and differences between P2P and MANET motivate an interesting and challenging research topic of enabling efficient $\mathrm{P} 2 \mathrm{P}$ file-sharing over MANET. The major problem is how to quickly find the requested file in spite of the mobility and the scarcity of power and bandwidth in the underlying MANET. This paper proposes five routing approaches combining existing $\mathrm{P} 2 \mathrm{P}$ searching protocols and MANET routing protocols. The performance of these approaches are evaluated and compared in terms of routing complexity, scalability, implementation complexity, maintenance complexity, energy efficiency, and so on. It is concluded that the crosslayer protocols perform better than simply overlaying $\mathrm{P} 2 \mathrm{P}$ protocols on MANET. The paper is organized as follows. Section 2 briefly reviews related works. Section 3 introduces the proposed approaches in details. The conclusion is drawn in section 4 .

\section{Related works}

Both P2P network and MANET are becoming popular only in the recent years. The $\mathrm{P} 2 \mathrm{P}$ file-sharing over MANET is still in its early stage. Klemm ([5]) presented a P2P system for searching and file transfer tailored to both the characteristics of MANET and the requirements of $\mathrm{P} 2 \mathrm{P}$ file sharing. We will see that the discovery protocol in this approach is similar to the first approach in this paper, which can be greatly improved by other proposed approaches. Hu ([6]) suggested a MANET routing protocol DPSR, which applies Pastry to a MANET routing protocol, DSR. This is because that Pastry can find requested information more efficiently (namely $O(\log n)$ ) than the regular MANET on-demand routing protocols. But how efficient is the new network routing protocol and how to integrate $\mathrm{P} 2 \mathrm{P}$ file-sharing applications to DPSR are still in need of further investigations. Siddhartha ([7]) proposed a P2P data dissemination protocol over an ad hoc network, where a file is divided into segments for the efficiency of transmission over a particular mobility model. But the issue of data discovery was not discussed, which will be the major topic in this paper. Papadopouli ([8]) introduced a peer-to-peer architecture called 7DS, which enables resource sharing in a self-organizing, peer-to-peer fashion without the need of an infrastructure. But its emphasis is on the application layer rather than on network routing protocols. Proem ([9]) is a P2P Platform for developing mobile P2P applications. Proem Mobile supports 802.11b in ad hoc mode. But Proem does not consider the multi-hop mobile ad hoc networks.

\section{P2P file-sharing over MANET}

There have been many routing protocols in $\mathrm{P} 2 \mathrm{P}$ networks and MANET respectively. Specifically, there are broadcast-based and DHT-based algorithms for P2P file searching, while most on-demand routing protocols in MANET are based on broadcast. We here introduce five approaches to integrate these protocols in different ways. Due to the space limit, we will only focus on route discovery in this paper, while the route maintenance will be extended in a future paper.

\subsection{Broadcast over broadcast}

A straightforward approach is to simply put broadcast-based P2P file lookup protocol over MANET on-demand network routing protocols. Specifically, the file request message at application layer will be broadcasted to every virtual neighbor peer in the P2P overlay. In order to get the source route to each virtual neighbor, the network routing 
request is also broadcasted at network layer. Assuming a peer $\mathrm{A}$ is searching a file stored at peer $\mathrm{B}$, the path taken by the request messages is illustrated by Figure 1 , where the square represents peer in P2P overlay and the dot represents mobile peer in MANET. The thick and thin lines represent routing paths at application layer and network layer, respectively. The shortest path from source A to destination B is drawn in dashed line.

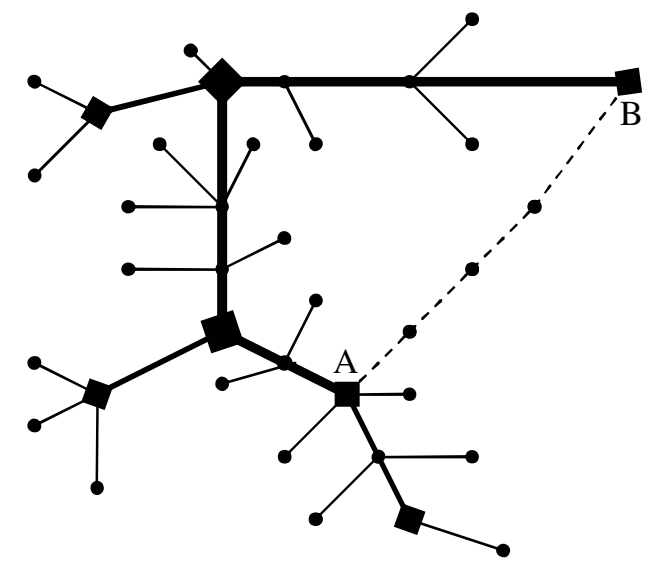

Figure 1. Broadcast over broadcast

This approach is easy to be implemented. But it suffers from the scalability problem due to the double broadcasts, which also incur a lot of energy consumption because every peer should always be aware of requests from all the other peers. In addition, the resultant path taken by requesting messages is not the shortest path between the source and destination because the virtual neighbors in P2P overlay might be physically far away from each other. The complexity of this routing algorithm is $O\left(n^{2}\right)$. All these issues make this approach only work temporarily for small MANET.

\subsection{Broadcast}

Due to the fact that wireless networks usually employ broadcast to send data even for unicast packets. The network routing protocols can be skipped when the application packets from the P2P searching protocol are required to be broadcasted. In order to further simplify the interaction between application layer and network layer, the virtual neighbors in the P2P overlay can be directly mapped to the physical neighbors. The path taken by the route request messages is displayed in Figure 2.

This approach removes the virtual overlay in conventional P2P file-sharing systems in order to facilitate direct interaction between applications and underlying wireless networks. The advantages of this approach are that it is simple to be implemented and the shortest path is obtained. But due to the nature of broadcast, the whole network is still flooded by the requesting messages, which imposes heavy burden on communication bandwidth and power supply for every mobile node. The complexity of this routing algorithm is $O(n)$. So this approach is more scalable than the first approach, but sill cannot work for large networks.

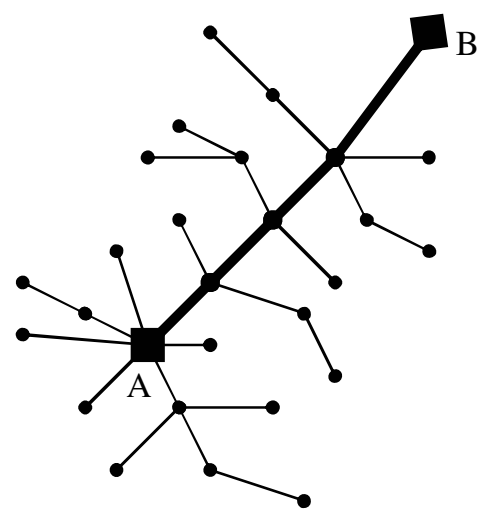

Figure 2. Broadcast

\subsection{DHT over broadcast}

Since the recently invented DHT-based searching protocols make the $\mathrm{P} 2 \mathrm{P}$ file-sharing networks much more scalable, we can directly apply a DHT-based P2P protocol on top of MANET. To this end, every file name and peer ID is hashed to a key by standard hash algorithms. Every peer should maintain a small routing table of size $O(\log n)$, in which each entry directs to an intermediate peer closer to the requested key. The peer closest to the requested key knows the address of the actual peer storing the requested file. But in order to get to these intermediate peers, we still need underlying network routing protocols which are usually based on broadcast in MANET. Figure 3 shows the routing path by this approach, where the square represents the peer in P2P overlay that is on the routing path by DHT-based protocol.

The DHT-based protocols introduce complexity in implementation. In order to maintain the correctness of each routing table, peers should communicate to each other by some stabilization protocols periodically. These protocols should be triggered more frequently for MANET due to mobility in underlying physical networks. An additional neighbor table involving peers with the nearest keys may also be needed to improve the robustness. Even more, every communication between two peers in $\mathrm{P} 2 \mathrm{P}$ overlay 
involves broadcast-based network routing in MANET network layer in order to get the source route. According to Figure 3, the complexity of this routing algorithm is $O(n \log n)$, which is surprisingly even higher than the Broadcast approach in subsection 3.2. This is due to the fact that network routing protocols in MANET introduce complexity of $O(n)$ to find the route between every two peers, although there are only $O(\log n)$ peers needed in the $\mathrm{P} 2 \mathrm{P}$ overlay.

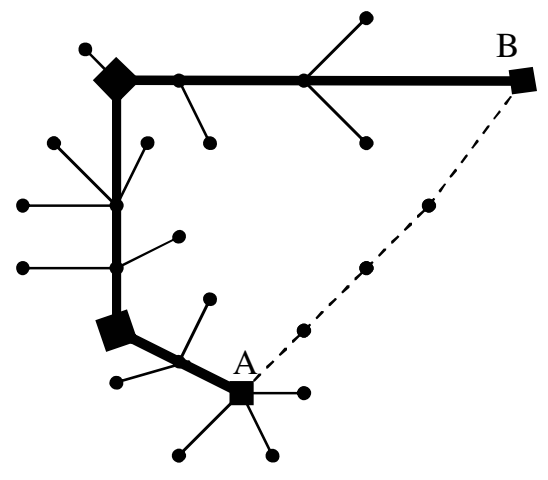

Figure 3. DHT over broadcast

\subsection{DHT over DHT}

Most current on-demand routing protocols in MANET adopt broadcast to propagate route requests. This significantly affects the scalability of the above approaches. On the other hand, the DHT-based P2P lookup algorithms greatly speed up information discovery in P2P networks. Due to the similar selforganizing nature of $\mathrm{P} 2 \mathrm{P}$ and MANET, it is possible to apply DHT to the MANET routing protocols so as to improve the network routing performance ([6]). In the circumstance of P2P file-sharing over MANET, one should further consider how to integrate the DHTbased P2P searching protocol to the underlying DHTbased MANET routing protocol. In this subsection, we consider the simple case when DHT is employed by P2P overlay and MANET separately. Specifically, for the DHT-based network protocol in MANET, the network ID (such as IP address) of every mobile node is hashed to a key and is evenly distributed across the network. For every entry in a peer A's routing table, a peer B's key and the route from A to B are stored. Since a key corresponds to a mobile node's network ID, a request for the route to this mobile node is guaranteed to reach the requested node as far as the DHT algorithm is correctly executed. Figure 4 displays the routing path, where the dotted line represents the shortest path between peers in $\mathrm{P} 2 \mathrm{P}$ overlay, while the solid line represents the actual network route in MANET.

The DHT over DHT approach introduces implementation complexity at both P2P overlay and network layer. The routing table in the DHT-based routing protocol should be further updated in case the route is changing or the peer is moving. The significance of adopting DHT algorithms is the improvement of scalability. According to Figure 4, the complexity of this routing algorithm is $O\left((\log n)^{2}\right)$, which is much better than previous approaches. For the power consumption, mobile nodes using DHTbased routing protocols can go to the 'sleep' mode periodically so that this approach can be made more energy-efficient than previous approaches. But on the other hand, the mobility of peers requires frequent update of routing tables and neighbor tables at both P2P overlay and MANET network layer.

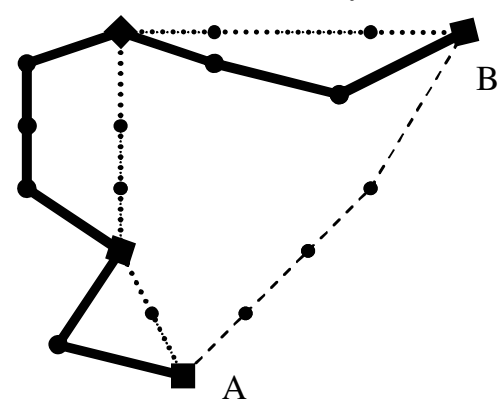

Figure 4. DHT over DHT

\subsection{DHT}

In order to reduce the implementation complexity in the DHT over DHT approach, we introduce a single cross-layer DHT routing protocol which can process both file requests and network route requests. To this end, both the file name and network ID are hashed to the same key space. When a new file is added, its key is stored at the peer with the closest key value to this file's key. Each entry in the routing table contains a pair of key and route. The routes can be initialized by broadcast. After that, the table will be periodically updated. By the lookup algorithm of DHT, a request for the route to a mobile node is guaranteed to reach the requested node in $O(\log n)$ steps, while a request for a particular file will be directed to the peer with the closest key to the key of this file in $O(\log n)$ steps. So the overall routing complexity is $O(\operatorname{cog} n)$ in terms of hops, where $c$ is a parameter representing the average number of hops in one lookup step. The routing path is illustrated in Figure 5, where the big 
dot represents mobile node in each lookup step and the little dot represents the intermediate mobile node in every step.

Similar to the Broadcast approach in subsection 3.2, this DHT approach removes the virtual P2P overlay so that the application-layer file search is integrated to the network-layer routing. In contrast to the DHT over DHT approach, this cross-layer approach simplifies the implementation and provides a more efficient routing protocol.

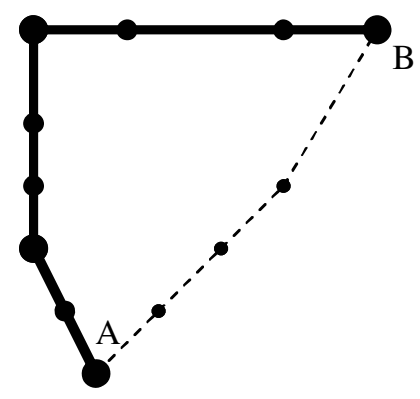

Figure 5. DHT

\section{Summary}

In this paper, we investigate $\mathrm{P} 2 \mathrm{P}$ file-sharing over MANET. Five routing protocols of different complexity are proposed. The comparison is given in Table 1, in terms of routing complexity, scalability, implementation complexity, maintenance complexity, energy efficiency, the shortest path and cross-layer property.

Table 1. Comparison of approaches

\begin{tabular}{|l|c|c|c|c|c|}
\hline & $\mathbf{3 . 1}$ & $\mathbf{3 . 2}$ & $\mathbf{3 . 3}$ & $\mathbf{3 . 4}$ & $\mathbf{3 . 5}$ \\
\hline Routing & $\mathrm{O}\left(n^{2}\right)$ & $\mathrm{O}(n)$ & $\mathrm{O}(n \log n)$ & $\mathrm{O}\left((\log n)^{2}\right)$ & $\mathrm{O}(\log n)$ \\
\hline Scalability & Bad & $\mathrm{Bad}$ & $\mathrm{Bad}$ & Good & Excellent \\
\hline Implementation & Low & Low & Medium & High & Medium \\
\hline Maintenance & Low & Low & Medium & High & Medium \\
\hline $\begin{array}{l}\text { Energy } \\
\text { efficiency }\end{array}$ & Low & Low & Low & Medium & Medium \\
\hline $\begin{array}{l}\text { The Shortest } \\
\text { Path }\end{array}$ & No & Yes & No & No & No \\
\hline Cross-layer & No & Yes & No & No & Yes \\
\hline
\end{tabular}

Conclusions are drawn as follows.

- The cross-layer design coordinates P2P protocols at application layer and routing protocols at network layer, which offers significant performance improvement in Broadcast and DHT approach.

- The Broadcast approach can be easily implemented for MANETs of small size.

- DHT approach is scalable to large networks. But its routing table and neighborhood table need to be carefully maintained. The proposed approaches apply to any DHT-based algorithms, such as Chord, Pastry, Tapestry and CAN.

\section{References}

[1] http://gnutella.wego.com.

[2] H. Balakrishnan, F. Kaashoek, D. Karger, R. Morris and I. Stoica, "Looking up Data in P2P Systems", Communications of the ACM, 46(2), Feb. 2003, pp. 43-48.

[3] E. M. Royer and C.-K. Toh, "A Review of Current Routing Protocols for Ad Hoc Mobile Wireless Networks", IEEE Personal Communications Magazine, 6(2), 1999, pp. 46-55.

[4] R. Shollmeier, I. Gruber and M. Finkenzeller, "Routing in Mobile Ad Hoc Networks and Peer-to-Peer Networks, a Comparison", Inter. Workshop on Peer-to-Peer Computing, Pisa, Italy, May 2002.

[5] A. Klemm, C. Lindemann and O. Waldhorst, "A SpecialPurpose Peer-to-Peer File Sharing System for Mobile Ad Hoc Networks", Proc. Workshop on Mobile Ad Hoc Networking and Computing, Sophia-Antipolis, France, March 2003, pp. 41-49.

[6] Y. C. Hu, M. D. Saumitra and H. Pucha, "Exploiting the Synergy between Peer-to-Peer and Mobile Ad Hoc Networks", Proc. of HotOS-IX, Hawaii, May 2003.

[7] K. G. Siddhartha, M. Singh, D. Xu, and B. Li, "Efficient Peer-to-Peer Data Dissemination in Mobile Ad-Hoc Networks", Proc. of International Workshop on Ad Hoc Networking, Vancouver, BC, August 2002.

[8] M. Papadopouli, and H. A. Schulzrinne, "Performance Analysis of 7DS a Peer-to-Peer Data Dissemination and Prefetching Tool for Mobile Users", Advances in wired and wireless communications, IEEE Sarnoff Symposium Digest, Ewing, NJ, March 2001.

[9] G. Kortuem, et al., "When Peer-to-Peer Comes Face-toFace: Collaborative Peer-to-Peer Computing in Mobile Adhoc Networks", Inter. Conference on Peer-to-Peer Computing, Linköpings, Sweden, August 2001. 